\title{
Kernos
}

Revue internationale et pluridisciplinaire de religion grecque antique

7| 1994

Varia

\section{Paradoxographie et religion}

\section{Manuel García Teijeiro et Maria Teresa Molinos Tejada}

URL : http://journals.openedition.org/kernos/1112

DOI : $10.4000 /$ kernos. 1112

ISSN : 2034-7871

\section{Éditeur}

Centre international d'étude de la religion grecque antique

Édition imprimée

Date de publication : 1 janvier 1994

ISSN : 0776-3824

\section{Référence électronique}

Manuel García Teijeiro et Maria Teresa Molinos Tejada, « Paradoxographie et religion », Kernos [En ligne], 7 | 1994, mis en ligne le 20 avril 2011, consulté le 22 avril 2019. URL : http:// journals.openedition.org/kernos/1112 ; DOI : 10.4000/kernos.1112 
Kernos , 7 (1994), p. 273-285.

\section{PARADOXOGRAPHIE ET RELIGION}

Dès le III siècle av. J.-C., un goût passionné pour les collections se développe dans le monde ancien. Les parémiographes, les doxographes, les glossographes, les mythographes, etc., recueillent des proverbes, des maximes, des gloses, des mythes. Les paradoxographes ${ }^{1}$ se sont spécialisés dans la compilation de paradoxes : faits étonnants, contraires à l'opinion commune, à ce que l'on pourrait s'attendre. Ils publient de véritables recueils de petits textes, généralement brefs, des notices étranges, juxtaposées, sans trop de lien entre elles, qu'ils trouvent éparses dans les œuvres d'auteurs précédents. L'établissement des grandes bibliothèques facilite la tâche. Il n'est pas surprenant que le premier exemple connu d'une telle collection soit précisement le recueil de Callimaque, bibliothécaire d'Alexandrie.

Les paradoxographes répondent à la demande du grand public hellénisé, avide de lire, mais très peu exigeant et critique, et dont les goûts se transforment graduellement, comme le démontre l'évolution même de la paradoxographie : les notices de Callimaque ne sont pas comparables aux prodiges sensationnels de Phlégon de Tralles. Délimiter le sujet et les thèmes des mirabilia est une opération difficile puisque le type même de ces collections permet aisément les intrusions les plus variées, mais on peut, de toutes façons, affirmer que, au moins au début, c'est la nature qui est leur champ d'enquête : les merveilles des eaux, des pierres, des plantes, des animaux, puis de l'homme. Les paradoxographes ne sont donc pas ni des mythographes, comme l'est Ptolémée Chennos, ni des auteurs rationalistes, comme, par exemple, Palaiphatos.

Grâce aux paradoxographes, de nombreuses informations ont été conservées : notices ethnographiques, géographiques, littéraires, cultuelles, religieuses, croyances populaires pleines de superstitions... Ils rapportent parfois un fait que nulle autre source, grecque ou latine, ne fournit. D'où leur intêret et leur importance. C'est précisément le cas de

1 A. Westermann, Paradoxographi Graeci, Braunschweig, 1839; A. Giannini, Paradoxographorum Graecorum reliquiae, Milano, 1965. Cf. aussi O. KELLER, Rerum naturalium scriptores Graeci minores, I, Leipzig, 1877. 
l'anecdote $43 \mathrm{du}$ Paradoxographe de Florence ${ }^{2}$, auteur anonyme d'un recueil de merveilles des eaux, dont on n'en finit pas d'établir la date avec exactitude, peut-être écrit au premier siècle de notre ère.

La notice est la suivante: "En Lydie se trouve un lac nommé Tala qui est consacré aux nymphes. Il produit une foule de roseaux et parmi eux l'un à qui les indigènes donnent le titre de roi. Ils le supplient en célébrant des sacrifices et des fêtes annuelles. Lors de leur célébration, lorsqu'éclate sur le rivage le bruit d'un concert, tous les roseaux se mettent à danser et le roi, qui danse avec eux, arrive sur le rivage. Les indigènes le couronnent de bandelettes avant de le renvoyer, souhaitant se retrouver ensemble la prochaine fois. Ceci est un signe de prospérité. Tel est le récit d'Isigonos dans le second livre de ses Choses Incroyables».

Est-ce que ces roseaux dansants, ce roi des roseaux, sont de simples fantaisies du paradoxographe ? La précision de détails indiquent plutôt le contraire, et, bien que ni le couronnement ni la cérémonie soient ailleurs mentionnés, d'autres auteurs anciens parlent aussi de ce lac de la Lydie en ajoutant des détails supplémentaires qui complètent notre information.

Varron, Pline, Strabon, Sénèque ${ }^{3}$ confirment l'existence en Lydie, à 40 stades au nord de Sardes, d'un lac marécageux, rempli de joncs et de roseaux, ainsi que d'îles flottantes. Cet étang, où se déroulaient des fêtes

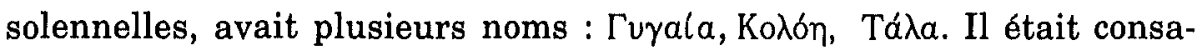
cré aux nymphes et détenait une grande sainteté. Sans aucun doute, ces auteurs font tous référence à un même endroit, l'actuel lac-marécage de Mermère, situé et décrit avec précision par L. Robert dans ses Documents d'Asie Mineure ${ }^{4}$, où il inclut des reproductions de cartes et des photos.

Tous ces témoignages anciens sur le lac de la Lydie ont un point commun : ils y attestent la présence d'îles flottantes, phénomène tout à fait réel, car il s'agit d'agglomérats de matières végétales non fixés au fond, donc susceptibles de dériver, et suffisamment compacts pour que s'y installent des roseaux et d'autres plantes de marais. Ce phénomène

2 Édition et commentaire de H. OEHLER, Paradoxographi Florentini anonymi opusculum de aquis mirabilibus, Tubingae, 1913.

3 VARron, De re rust., III 17, 4 et apud MART. CAP., IX, 928; SÉNEQUE, Nat. quaest., III, 25, 7; PLINE, Nat. hist. , II, 209; XXXI, 25; STRABON, XIII, 4, 5.

$4 \quad B C H, 106(1982)$, p. 334-359. 
n'est pas $d u$ tout exceptionnel, puisqu'on connaît son existence dans d'autres endroits marécageux du monde ancien, comme à Orchomène en Béotie, en Italie, dans le Delta en Égypte, etc. La mobilité de ces îles est très grande, car la plus légère brise ${ }^{5}$ suffit à les déplacer. Il y a, bien sûr, d'autres moyens, comme les perches dont nous parle Pline (Nat. hist,. II, 209) à propos justement des îles lydiennes qui furent le salut de beaucoup de citoyens romains lors de la guerre de Mithridate.

Varron et Pline $^{6}$ ajoutent un nouveau trait au tableau : ces petites îles du lac des nymphes "dansent»: au rythme de la musique elles se déplacent en décrivant des cercles, de la rive jusqu'au centre de l'étang, pour revenir ensuite au bord. On emploie même le verbe grec $\chi \circ \rho \in v ́ \epsilon \iota \nu$ pour décrire ces mouvements et on nous donne leur nom : elles sont appelées saliares, "les sauteuses». Il s'agit, sans doute, d'une cérémonie religieuse, que Varron, dont on sait qu'il fut un grand voyageur, affirme avoir vu de ses propres yeux. Le nom des îles, saliares, et la description de la musique dont la mélodie provoque leur mouvement, évoquent l'idée d'un chant rythmique appuyé par le frappement répété des pieds dans certaines danses rituelles.

D'après ces informations anciennes nous avons, donc, à l'étang de nymphes, en Lydie, des îles mobiles qui dansent au rythme d'un cantus. Or Strabon introduit une légère modification. Tout en se méfiant de la

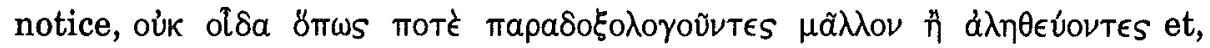
en même temps, révélant involontairement sa source, évidemment un paradoxographe, il affirme que ce qui dansait sur ce lac était des кá $\lambda \alpha \mu \iota^{7}$, des roseaux. La coïncidence de ses paroles avec celles $d u$ paradoxographe est frappante. Strabon, sceptique, résume en une seule phrase la cérémonie et sans la description plus étendue de notre paradoxographe nous n'aurions pas su davantage.

En combinant, donc, tous ces témoignages, les îles dansent tout comme le font les roseaux du lac. Comment interpréter ces textes ? Il est évident que les roseaux n'effectuent pas de mouvement et qu'ils ne peuvent pas danser. Il faudrait, à présent, tenir compte des traits carac-

5 SÉNÈQUE, Nat. quaest. , III, 25, 8.

6 Loc. cit.

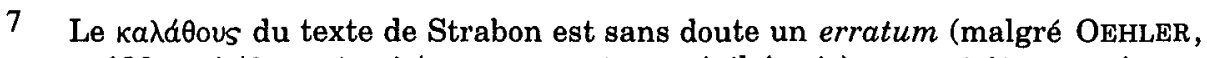
p. $120: \kappa a \lambda \alpha \theta_{0}$ ovs et $\kappa a \lambda \alpha \mu_{\mu}$ ovs ne sont pas si éloignés) ou peut-être aussi une tentative de rationaliser la notice sans trop y croire, comme l'appréciation critique finale le suggère. Le texte du paradoxographe garantit la correction. Cf. L. ROBERT, loc. cit., p. 350 sq. 
téristiques de ces collections de paradoxes et de leurs techniques particulières pour la fabrication du merveilleux ${ }^{8}$.

D'une part, les paradoxographes prétendent que soit fiable et crédible ce qu'ils racontent. D'où leurs efforts pour situer exactement l'emplacement géographique, le lieu où se produit la merveille (il ne s'agit pas d'une pure fantaisie; on peut, si l'on veut, la vérifier). Dans notre cas, la Lydie, plus précisément le lac Tala, consacré aux nymphes. Et, comme en règle générale, les merveilles sont tirées de l'œuvre d'autres auteurs, ils consignent soigneusement la provenance, non seulement de

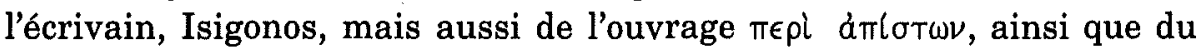
livre, le second.

D'autre part, bien qu'ils prétendent être sérieux, précis, «scientifiques», recourant à certains procédés caractéristiques (simplification du texte original, adaptation, omission de toute explication), ils fabriquent dans une certaine mesure la merveille. On peut aisément reconnaître leurs méthodes quand la source de la notice paradoxographique nous est, elle aussi, parvenue. Par exemple, l'anecdote 80 d'Antigonos et l'Histoire des animaux, IV, 11 d'Aristote. Celui-ci étudie longuement le sexe de l'anguille. Ce texte est résumé en une seule phrase par Antigonos : «L'anguille n'est ni de sexe masculin, ni de sexe féminin». Ainsi formulé, sans argumentation, sans discussion, sans aucune explication, le problème est beaucoup plus étonnant et singulier. Il devient un paradoxe.

Malheureusement dans notre cas, nous ne conservons ni l'œuvre d'Isigonos, ni ses sources, mais en extrapolant le procédé, on peut logiquement déduire qu'il ne s'agissait pas de roseaux dansants, mais probablement d'un chœur paré, ou peut-être déguisé, de joncs ${ }^{9}$ qui dansait. L'étape suivante, qui est celle d'affirmer que c'était les roseaux

8 Cf. Chr. JACOB, Sur la paradoxographie grecque, in Lalies, 2 (1983), p. 121-140.

9 Dans le monde grec ancien on connaît plusieurs danses mimétiques, cultuelles, dans lesquelles les exécutants se déguisaient ou simplement se paraient de certains attributs animaux ou végétaux. Cf. K. LATTE, De saltationibus Graecorum armatis, Königsberg, 1913, et, plus récemment, L.B. LAWLER, The Dance in Ancient Greece, Univ. of Iowa, 1964. Dans notre cas, il s'agit certainement de la personnification de quelque esprit de la végétation. FRAZER dans son livre The Golden Bough offre de nombreux exemples de personnifications similaires au moyen de couronnes de fleurs, de feuilles, etc. Souvent la personne ainsi déguisée est appelée «roi»: cf. les "rois de mai», qui sont couronnés de guirlandes. 
qui dansaient, est facile à franchir et rentre parfaitement dans leurs méthodes.

Notre paradoxographe, donc, décrit pour la première et unique fois, les célébrations religieuses du lac lydien. Ses mots éveillent l'ambiance de tant d'hymnes conservés dans lesquels on invoque la présence de la divinité, où on la prie de se montrer bienveillante et où on finit par la congédier, souhaitant se retrouver à nouveau, au même endroit. On se souvient, par exemple, des derniers mots adressés à Adonis, figure associée aussi à la végétation, dans la quinzième idylle

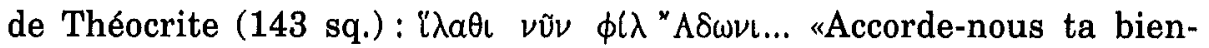
veillance maintenant, cher Adonis, et garde-la nous pour l'an prochain; maintenant nous t'avons accueilli avec joie, Adonis; et lorsque tu reviendras, nous t'accueillerons amicalement».

Le récit du paradoxographe conserve le témoignage d'une propitiation rituelle d'une divinité de la végétation et plus précisément d'un rite pour la prospérité des $\kappa a ́ \lambda \alpha \mu o l$, des roseaux, si importants pour les gens qui exploitent ces lacs, aujourd'hui comme jadis. Les roseaux abritent une foule d'oiseaux migrateurs, que l'abondance de poissons du lac attire (pêche et chasse sont deux ressources très importantes de la vie

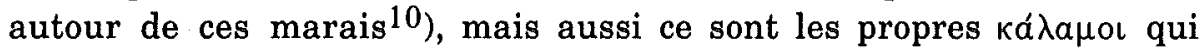
constituent une richesse de premier rang; roseaux dont les habitants se servaient autrefois, et encore aujourd'hui, à de nombreuses fins : fabrication de nattes, qu'ils commercialisent et exportent et qui sont indispensables aussi bien dans les maisons que pour sécher les poissons au soleil, ou même pour la construction de logis, pour la couverture de murs et de toitures, etc. L. Robert fournit dans l'article ci-dessus mentionné des informations très intéressantes au sujet de l'exploitation traditionnelle de ces lacs à l'heure actuelle. Ces observations font ressortir

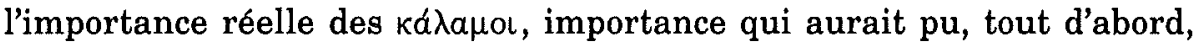
nous échapper. Donc, on ne s'étonne plus de l'existence de rites pour la prospérité des roseaux; rites que nous n'aurions pas connus sans la notice du Paradoxographe de Florence.

Voyons encore quelques exemples de l'intérêt que la paradoxographie peut offrir à l'étude de la religion. Nous avons déjà mentionné les histoires à sensation de Phlégon de Tralles, esclave affranchi de l'empereur Hadrien. Nous possédons trente-cinq de ses récits qui parlent exclusivement de l'homme: contes de revenants, cas de

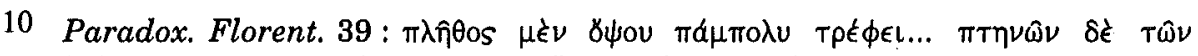

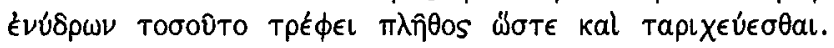


changement de sexe, narrations sur des hermaphrodites, découvertes d'ossements gigantesques, naissances prodigieuses, etc. Les trois premiers, qui portent sur des fantômes, contiennent certains différences de style très marquées par rapport aux autres, ce qui trahit une source différente dont les particularités ont été respectées par le paradoxographe ${ }^{11}$. Le plus précieux du point de vue littéraire est, sans doute, le premier, celui qui inspira à Gœthe sa Braut von Korynth. C'est l'histoire d'une fille ${ }^{12}$ décédée peu après son mariage, qui, pendant la nuit, visite un jeune hôte chez ses parents; celui-ci ne se méfie pas, la reçoit de bon gré et tous les deux jouissent de leurs amours pendant trois nuits, jusqu'au jour où le fantôme est découvert et meurt définitivement.

Il existe des récits semblables à des époques et des lieux très différents $^{13}$, preuve que ce conte est fondé sur un sujet folklorique très répandu. Dans la version grecque n'apparaissent pas les traits vampiriques que l'on découvre ailleurs. Il est plus proche du thème de l'union entre un mortel et un être de l'au-delà; union brisée aussitôt qu'on découvre la vraie nature du personnage. Phlégon néanmoins met fin à son histoire en signalant que le jeune homme, dont la fille fantôme était tombée amoureuse, se suicida peu après plein d'appréhension, ce qui semble une tentative de rationaliser une vieille croyance : le contact avec un mort entraîne toujours la mort.

Du point de vue de la religion, il est aussi très intéressant que le récit rapporte les prescriptions d'un devin recommandant porter le cadavre au-delà des frontières et de le brûler, tout en offrant des sacrifices à certaines divinités pour se les rendre propices. Ces mesures rappellent de près celles de Tirésias dans la XXIVe idylle de Théocrite ${ }^{14}$ pour se

11 Cf. J. MESK, Ueber Phlegons Mirabilia I - III, in Philologus, 80 (1925), p. 298311.

12 Le commencement de la narration manque dans le seul manuscrit existant, Cod. Palat. Graec. 398, mais on peut en reconstruire l'essentiel grâce à une notice de Proclus (Ad Remp., II, p. 116, 2 sq. Kroll). Cf. E. RoHDE, Zu den Mirabilia des Phlegons, in Kleine Schriften, Tübingen und Leipzig, 1901, p. $173-185$ (= RhM, 32, 1877, p. 329 sq.).

13 Cf. W. F. Hansen, An Ancient Greek Ghost Story, in Folklore on two Continents. Essays in Honor of L. Dégh, Bloomington, 1980, p. 71-77, et Contextualizing the Story of Philinnion, in Midwestern Folklore, 15 (1989), p. 101-108.

14 XXIV, 88 sq. Cf. Gow, ad. loc. Dans le poète hellénistique, cependant, il faut brûler les serpents et puis jeter la cendre à un fleuve, afin que le cours d'eau la porte au-delà des frontières. 
défaire des deux serpents qui avaient attaqué le petit Héraclès pendant la nuit.

La seconde et la troisième histoire de Phlégon sont des contes de revenants où des oracles ont été insérés. La seconde rapporte un prodige qui se serait déroulé en Étolie ${ }^{15}$. Policrite, premier magistrat du pays, s'étant marié avec une femme locrienne, mourut quatre jours après les noces. Neuf mois plus tard, sa veuve mit au monde une affreuse créature hermaphrodite. La famille, terrifiée, appela l'assemblée du peuple pour délibérer et prendre une décision. Alors les prêtres et les devins furent consultés. Certains affirment que le prodige annonce de graves dissensions entre Étoliens et Locriens. D'autres conseillent l'expulsion de la mère et du fils, ainsi que leur destruction par le feu. À ce moment-là, le fantôme de Policrite, habillé en noir, fit son apparition et demanda à l'assemblée de lui rendre son fils, car il ne permettrait jamais son anéantissement. Comme ils restaient hésitants et frappés de stupeur, le fantôme reprit la parole pour décliner toute responsabilité quant aux terribles conséquences que leur refus pourrait provoquer. Il prit son fils dans ses mains et, sans faire cas des cris et des jets de pierre de la foule effrayée, il avala le petit, épargnant seulement la tête, et aussitôt disparut. Les citoyens terrifiés, ne sachant quel parti prendre, décidèrent d'envoyer une légation à Delphes afin de consulter l'oracle; mais la tête du petit, qui se trouvait à même le sol, produit un oracle en hexamètres leur interdisant de le faire, puisqu'ils avaient les mains souillées par le sang. Elle prédit des maux terribles qui allaient survenir au terme d'une année, lors d'une guerre funeste contre les Locriens et les gens d'Acarnanie.

Phlégon raconte ensuite une troisième histoire plus curieuse encore que la précédente ${ }^{16}$. Tout comme dans les autres narrations, il y a aussi un fantôme, celui d'un ßıаıodávatos, quelqu'un qui avait donc disparu de mort violente. La narration commence par la victoire remportée par Acilius Glabrio sur Antiochos le Grand aux Thermopyles (195 av. J.C.). Certains prodiges survenus poussent les Romains à envoyer une délégation à Delphes, où la Pythie les engage à freiner leur conquête, car la colère d'Athéna les menaçait de la ruine totale. Obéissant à ses ordres, les Romains renoncent à de nouvelles entreprises en Europe et rentrent à Naupacte où ils accomplissent des sacrifices publics dans le

15 Cf. L. BRISSON, Aspects politiques de la bisexualité. L'histoire de Polycrite, in Homm. à M. J. Vermaseren I, Leiden, 1978, p. 80-122.

16 Cf. A. PERETTI, Una storia di fantasmi oracolanti, in SCO, 33 (1983), p. 39-81. 
sanctuaire panhellénique. Pendant que les cérémonies se déroulent,

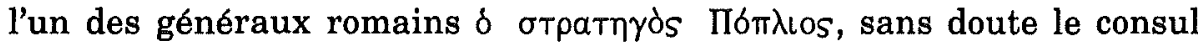
Publius Cornelius Scipio dont la réputation de devin était fort répandue ${ }^{17}$, entre subitement en transe et commence à prononcer des oracles, en prose et en vers, prophétisant la défaite romaine.

Après cela, il sort immédiatement du camp, suivi de près par une foule de soldats étonnés, et grimpe sur un chêne. Du haut de l'arbre, il annonce qu'un grand loup roussâtre va le dévorer ce même jour. Peu après, en effet, la bête fait son apparition. Publius descend de l'arbre et, devant tout le monde, est englouti par l'animal qui épargne la tête. Au moment où les soldats veulent l'ensevelir, elle prend la parole pour le leur interdire : personne ne doit la toucher. Elle continue à prédire le déclin de la puissance romaine, qui sera anéantie par une armée venant du fond de l'Asie. C'était Apollon qui l'annonçait, le même qui avait envoyé le loup et qui avait emmené Publius chez les Bienheureux. Phlégon finit son récit en signalant que toutes les prédictions s'étaient

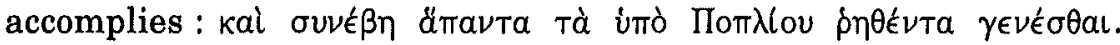

Évidemment, ces trois mirabilia sont des histoires étranges, dans les deux dernières le thème de l'apparition de revenants se mêlant avec celui des oracles. Ceux-ci ont attiré l'attention de philologues et d'historiens, intéressés surtout par les oracles du dernier récit, qui ont été étudiés dans le cadre de la propagande anti-romaine de cette époque ${ }^{18}$. Mais il y a d'autres aspects qui peuvent intéresser les spécialistes de la religion. À présent, nous voulons en souligner deux.

Le premier est celui des têtes parlantes. Aussi bien celle du petit hermaphrodite que celle de Publius commencent à parler dès que le reste de leur corps est dévoré et elles défendent leur auditoire de les enterrer, Ces deux histoires sont celles qui dans la littérature grecque nous

17 Cf. E. GABBA, P. Cornelio Scipione Africano e la leggenda, in Athenaeum, 53 (1975), p. 3-17.

18 Ainsi H. FUCHS, Der geistige Widerstand gegen Rom in der antiken Welt, Berlin, 1938, p. 5 sq.; H.W. PARKE - D.E.W. WORMELL, The Delphic Oracle, I, Oxford, 1956, p. 276 sq.; F. MARTELLI, In margine a un frammento di Antistene : FGrHist, 257, F 36, in RSA, 8 (1978), p. 123-131, et Ancora in margine ad un frammento di Antistene, ibid, 12 (1982), p. 251-260; J. D. Gauger, Phlegon von Tralleis, mirab. III. Zu einem Dokument geistigen Widerstandes gegen Rom, in Chiron, 10 (1980), p. 223-261; L. BREGLIA PULCI DORIA, Oracoli sibillini tra rituali e propaganda (Studi su Flegonte di Tralles), Napoli, 1983; D. S. POTTER, Prophecy and History in the Crisis of the Roman Empire, Oxford, 1990, p. 112. 
rappellent le mieux la tradition de la tête de Orphée, qui, transportée sur les eaux, chantait au son de la lyre et qui, touchant terre, se mit à rendre des oracles ${ }^{19}$.

Il est intéressant de constater que dans les deux récits de Phlégon les têtes font leurs prédictions immédiatement après que leurs corps sont dévorés, c'est-à-dire, après leur déchirement, comme le spécifient expli-

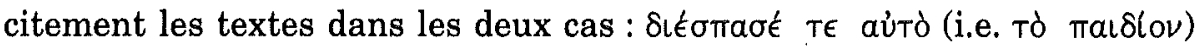

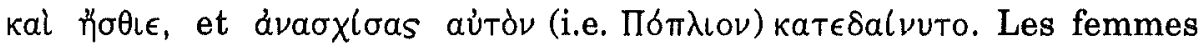
thraces déchirèrent aussi les membres d'Orphée. En fait, quelques témoignages anciens utilisent le même verbe que Phlégon : $\delta$ เađđá $\omega^{20}$.

On a cru voir dans la tête parlante d'Orphée un trait de chamanisme de ce personnage mythologique ${ }^{21}$. Les histoires du paradoxographe auraient gardé le souvenir d'un type d'oracle très ancien : une tête humaine, qu'ensuite l'oracle d'Apollon aurait remplacée ${ }^{22}$. D'ailleurs, il faut considérer que le motif des têtes coupées parlantes est fort répandu. On en a des témoignages à des endroits et à des époques diverses. Il est spécialement fréquent dans le monde celtique, où ces têtes ont reçu un véritable culte ${ }^{23}$; on le retrouve dans la mythologie norroise, dans la tête décapitée du géant Mimir, que Odinn oignit d'herbes et enchanta de telle façon qu'elle lui parla de beaucoup de choses secrètes ${ }^{24}$. Le motif réapparaît aussi dans l'hagiographie chrétienne et a donné lieu à plusieurs traditions populaires sur la fabrication de têtes artificielles, le plus souvent en bronze, capables de parler et de prédire l'avenir, dont la plus célèbre est, sans doute, celle de Saint Albert le Grand. On en rencontre beaucoup d'autres, par exemple dans un épisode de Don Quijote (II 62). Il serait facile d'en multiplier les exemples, mais à présent nous voudrions attirer l'attention sur ceux que

19 Cf. récemment M. ScHMIDT, Ein neues Zeugnis vom Orpheushaupt, in AK, 15 (1972), p. 128-137; F. GRAF, Orpheus : A Poet among Men, in Interpretations of Greek Mythology, London, 1987, p. 92-106 ; J. DoERIG, La tête qui chante, in Orphisme et Orphée, en l'honneur de J. Rudhardt, Genève, 1991, p. 61-64.

20 O. KeRN, Orph. Fragm. T 113 (Ps.-Ératosth.), 118 (Lucien).

21 Cf. la critique de J. N. BRemmer, The Early Greek Concept of the Soul, Princeton, 1983, p. 46 sq. (antérieurement Amsterdam, 1979, p. 32).

22 Ainsi W. DÉONNA, Orphée et l'oracle de la tête coupée, in REG, 38 (1925), p. 45.

23 Cf, par exemple, Cl. STERCKX, La tête et les seins. La mutilation rituelle des ennemis et le concept de l'âme chez les Celtes, Sarrebruck, 1975, et Les têtes coupées et le Graal, in Studia Celtica, 20-21 (1985-1986), p. 1-43.

Ynglinga Saga, chap. IV; Völuspá, 46; Sigrdrífumál, 14. 
l'on trouve dans les papyri magiques grecs, qui prouvent l'expansion de cette croyance superstitieuse à l'époque impériale. L'un d'eux (PGM, IV, 1928-2005) s'intitule : «Formule d'évocation du roi Pytis sur n'importe quel crâne». Un autre, qui suit immédiatement dans le manuscrit (2006-2125), a la forme d'une lettre, tout comme les mirabilia de Phlégon ci-dessus mentionnés. Elle s'ouvre par : "Pytis au roi Ostanès, salut. Puisque tu m'écris constamment sur la consultation des crânes... ${ }^{25}$ ". Dans les deux cas il s'agit d'enchantements pour obtenir l'aide d'un démon, qui par la suite deviendra à vie le serf du mage. Le crâne constitue la "matière magique», la oủol $\alpha^{26}$, pour évoquer l'esprit $d u$ mort, c'est-à-dire, le démon. Entre les deux recettes, ce même papyrus fournit une formule magique pour empêcher de parler les crânes qui ne sont pas appropriés pour la divination (PMG IV 2125-2139) ${ }^{27}$. Le codex astrologique de Milan ${ }^{28}$ préserve un document byzantin donnant des instructions pour préparer un crâne à répondre à toutes sortes de questions. Parmi ces préparatifs, on trouve un cercle magique et un chat noir, très courants dans les pratiques de la magie médiévale. Bien avant, vers le milieu du IIIe siècle après J.-C., le témoignage d'Hippolyte ${ }^{29}$ montre comment on pouvait fabriquer un crâne parlant avec de la cire, du cuir et un tuyau dissimulé à l'intérieur; on arrivait même à un coup de théâtre terrifiant : le crâne disparaissait sous les yeux des spectateurs quand la cire fondait à la chaleur des flammes d'une offrande.

Mais laissons en paix la magie noire et revenons à notre paradoxographe. L'autre aspect que nous voudrions faire remarquer ici se rapporte à l'apparition des spectres. Aussi bien dans la seconde que dans la troisième histoire on nous dit que le revenant parle $\lambda \epsilon \pi T n \tilde{n} T \tilde{n} \phi \omega \nu \tilde{n}$, "d'une voix faible». Nous avons là un témoignage très intéressant de la croyance qui attribue aux fantômes une voix étouffée, sans force.

Chez Homère, les âmes des morts émettent des bruits confus qui ne constituent pas un vrai parler. Pour se référer à ces voix le poète se sert

25 J. B IDEZ - F. CUMONT, Les mages hellénisés II, Paris, 1938, p. 308 ( $\mathrm{n}^{\circ} 28$ ).

26 Dans PGM, IV,1885 sq. on emploie comme oúola l'os de la tête de quelqu'un mort violemment.

27 Cf. T. HOPFNER, Griechisch-ägyptischer Offenbarungszauber, II, 2, Amsterdam, 1990, p. 421 sq. (nouvelle édition, plus commode que celle de Leipzig, 1924), mais okú申os signifie ici "crâne", non "vase".

28 Codd. astrolog., III. Appendix, p. 53. Cf. HOPFNER, op. cit., II, 2, p. 613 sq.

29 Refut. , IV, 41. Cf. HOPFNER, op. cit., II, 2, p. 616 sq. 
du verbe $\tau \rho l \zeta \omega$ qui s'applique aussi au piaillement plaintif des petits oiseaux ( $B, 314)$. Lorsque l'esprit de Patrocle se présente à Achille endormi, il lui parle de la même voix qu'il avait de son vivant $(\Psi, 67)$, mais quand, telle une fumée, il rentre sous terre, chez l'Hadès, il le fait en chuchotant ( $\tau \in \mathrm{T} \rho \mathbf{\gamma} v \tilde{\tau} \alpha, \Psi, 101$ ). Dans l'Odyssée, les âmes des prétendants de Pénélope s'envolent aux enfers émettant des petits cris ténus. Le poète les compare aux chauve-souris ${ }^{30}$.

Puisque la tradition épique conçoit les $\psi u \chi a l$ comme des images $(\epsilon l \delta \omega \lambda \alpha)$ de ce qu'elles furent en vie, il paraît naturel qu'on se représente leurs voix comme des copies affaiblies du parler humain. Des auteurs plus récents, Virgile, Lucien, Philostrate, Claudien, nous rapportent une idée identique ${ }^{31}$. Même Shakespeare dans un passage connu de Hamlet $(\mathrm{I}, 1,115)$ :

the sheeted dead

Did squeak and gibber in the Roman streets

À côté de cette croyance, il en existe une autre, selon laquelle les morts peuvent transmettre des messages et prédire des événements futurs. Chez Homère, nous l'avons déjà vu, on rencontre les deux idées. Postérieurement on les voit se confondre, ayant pour résultat que les morts parlent d'une manière sensée, mais d'une voix étouffée, faible. C'est précisément cette conception que nous trouvons dans notre paradoxographe et qu'attestent aussi des témoignages d'époque alexandrine et romaine. Par exemple, dans l'Alexandra de Lycophron (686 s.), Cassandre, qui se réfère à la Nekyia de l'Odyssée, prédit qu'Ulysse entendra

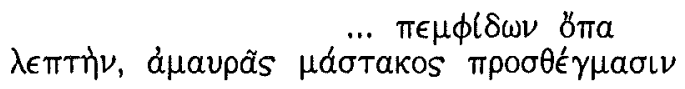

la voix ténue des ombres, émise par des lèvres languissantes.

Chez Ovide (Fast., V, 458) le fantôme de Rème transmet ses instructions exiguo murmure. Réciproquement, lorsqu'un nécromant prétend

$30 \omega 5$ et 9. Dans son commentaire A. Heubeck (Fondazione L. Valla, 1986) dit que le verbe, comme in $\Psi, 101$, «indica lo stridulo ronzare delle anime in volo, come si deduce anche della similitudine nei vv. 6-9". Le "piaillement" des morts, cependant, est attesté ailleurs. Cf. E.B. TYLOR, Primitive Culture, I, London 1871, p. 452; HOPFNER, op. cit., I, 191.

31 Virg., Aen., VI, 492 sq.; LuCien, Nec., 11; Phllostr., Imag., p. 300, 17 K.; ClaUd., In Ruf., I, 126; cf. aussi HoR., Sat., I, 8, 40 sq.; Hermès Trismégiste apud STOBÉE, I, 49, 44 (= XXIII, 33 Nock-Festugière). J.N. BREMMER, op. cit., p. 85 sq. 
réanimer un mort pour qu'il réponde à ses questions, il lui chuchote à l'oreille ses enchantements, comme la vieille égyptienne le fait dans les Éthiopiques d'Héliodore (VI, 14 sq.) : le cadavre finit par répondre d'une voix sourde, comme si elle sortait d'un gouffre ${ }^{32}$.

Le néoplatonisme rationalisa ces croyances tâchant d'expliquer que ce n'était pas un non-sens que les esprits puissent émettre des voix. Proclus oppose les âmes pures, qui s'élèvent par un mouvement rythmique en produisant des sons harmonieux, à celles qui ont eu une vie très attachée aux biens matériels, ont du mal à se séparer de leurs corps et errent sur ce monde émettant ces voix dépourvues de sens et ce

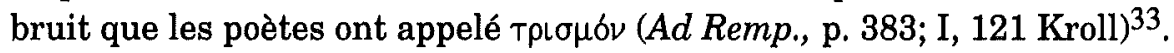

Parler bas, indistinctement, chuchoter ou murmurer, est une particularité des démons 34 . Dans certaines pratiques divinatoires qui s'effectuaient avec un bassin plein d'eau (lécanomancie), nous savons d'après les témoignages anciens qu'on entendait la voix des démons comme un sifflement ou un murmure à peine perceptible ${ }^{35}$. De même, le météorite qu'on utilisait dans un autre type d'oracles, semblable à l'actuel "oui-ja», produisait aussi un léger susurrement ${ }^{36}$.

Nous pouvons nous demander à quel point ces croyances au sujet de la façon de parler des esprits n'ont pas influencé la manière de prononcer les formules magiques, à voix basse, dans ces murmures dont les poètes et les sources littéraires nous parlent si souvent. Peut-être que la

32 «Il est souvent spécifié dans les textes que les formules d'évocation et de

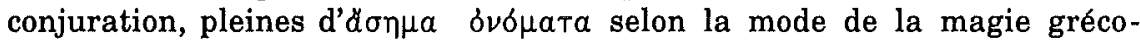
égyptienne, doivent être prononcées dans l'oreille de l'enfant qui sert de médium. À mon avis, cela signifie qu'elle doit être prononcée à voix basse", dit A. Delatte, $L a$ catoptromancie grecque et ses dérivés, Liège-Paris, 1932, p. 158 sq. $\AA$ ses témoignages on peut ajouter $P G M, I V, 2164-2166$. Cf. HOPFNER, op. cit., II, 2, p. 585; L. BALDINI MOSCADI, "Murmur" nella terminologia magica, in $S I F C, 48$ (1976), p. 254-262; M. GARCía T EIJEIRO, Recursos fonéticos y recursos gráficos en los textos mágicos griegos, in Revista Española de Lingüística, 19 (1989), p. 233-249.

33 HOPFNER, op. cit., I, p. 168 sq.

34 F. Bовнм, De symbolis Pythagoreis, Berlin, 1905, p. 33; K. SvoвоDA, La démonologie de Michel Psellos, Brno, 1927, p. 33 sq.

35 F. Bовнм, Hydromantie, in Handwörterbuch des deutschen Aberglaubens, IV, Berlin-Leipzig, 1932, col. 573, et Lekanomantie, ibid., V, col. 1207; A. DELATTE, op. cit., p. 73.

D AM., Vita Isidori, 203 (p. 276 Zintzen). Cf. HoPfNer, op. cit., II, 2, p. 510 sq. 
nécessité de garder secrets ces enchantements et, parfois aussi, le besoin d'impressionner le public, n'ont pas été les seuls motifs.

Laissons, cependant, cette question qui nous emmèneraient trop loin et concluons simplement en soulignant que ce sont les paradoxographes et leurs textes qui nous ont donné l'occasion de réfléchir sur des thèmes si divers : un rite pour la fertilité des roseaux, les têtes parlantes ou la diction des fantômes. Notre objectif était seulement d'insister sur le grand intérêt de la paradoxographie pour certains aspects de la religion ancienne.

M. García Teijeiro

Departamento de Filología Clásica

Ma.T. Molinos Tejada

Facultad de Filosofia y Letras

Universidad de Valladolid

E - 47002 V ALLADOLID 